EPJ Web of Conferences 71, 00007 (2014)

DOI: 10.1051/epjconf/20147100007

(C) Owned by the authors, published by EDP Sciences, 2014

\title{
Mass hierarchy and physics beyond the Standard Model
}

\author{
Ignatios Antoniadis ${ }^{1,2, a}$ \\ ${ }^{1}$ Department of Physics, CERN - Theory Division, $\mathrm{CH}-1211$ Geneva 23, Switzerland \\ ${ }^{2}$ On leave from CPHT (UMR CNRS 7644) Ecole Polytechnique, F-91128 Palaiseau
}

\begin{abstract}
I discuss the status of the mass hierarchy problem and prospects for beyond the Standard Model physics in the light of the Higgs scalar discovery at the LHC and the experimental searches for new physics. In particular, I will discuss in this context low energy supersymmetry and large extra dimensions with low string scale.
\end{abstract}

\section{Introduction}

During the last few decades, physics beyond the Standard Model (SM) was guided from the problem of mass hierarchy. This can be formulated as the question of why gravity appears to us so weak compared to the other three known fundamental interactions corresponding to the electromagnetic, weak and strong nuclear forces. Indeed, gravitational interactions are suppressed by a very high energy scale, the Planck mass $M_{P} \sim 10^{19} \mathrm{GeV}$, associated to a length $l_{P} \sim 10^{-35} \mathrm{~m}$, where they are expected to become important. In a quantum theory, the hierarchy implies a severe fine tuning of the fundamental parameters in more than 30 decimal places in order to keep the masses of elementary particles at their observed values. The reason is that quantum radiative corrections to all masses generated by the Higgs vacuum expectation value (VEV) are proportional to the ultraviolet cutoff which in the presence of gravity is fixed by the Planck mass. As a result, all masses are "attracted" to about $10^{16}$ times heavier than their observed values.

Besides compositeness, there are two main theories that have been proposed and studied extensively during the last years, corresponding to different approaches of dealing with the mass hierarchy problem. (1) Low energy supersymmetry with all superparticle masses in the TeV region. Indeed, in the limit of exact supersymmetry, quadratically divergent corrections to the Higgs self-energy are exactly cancelled, while in the softly broken case, they are cutoff by the supersymmetry breaking mass splittings. (2) TeV scale strings, in which quadratic divergences are cutoff by the string scale and low energy supersymmetry is not needed. Both ideas are experimentally testable at high-energy particle colliders and in particular at LHC.

On the other hand, the recent major discovery of the Higgs boson at the LHC with a mass around $126 \mathrm{GeV}$ is so far compatible with the Standard Model within $2 \sigma$ and its precision tests. It is also compatible with low energy supersymmetry, although with some degree of fine-tuning in its minimal version. Indeed, in the minimal supersymmetric Standard Model (MSSM), the lightest Higgs scalar

\footnotetext{
ae-mail: ignatios.antoniadis@cern.ch
} 
mass $m_{h}$ satisfies the following inequality:

$$
m_{h}^{2} \lesssim m_{Z}^{2} \cos ^{2} 2 \beta+\frac{3}{(4 \pi)^{2}} \frac{m_{t}^{4}}{v^{2}}\left[\ln \frac{m_{\tilde{t}}^{2}}{m_{t}^{2}}+\frac{A_{t}^{2}}{m_{\tilde{t}}^{2}}\left(1-\frac{A_{t}^{2}}{12 m_{\tilde{t}}^{2}}\right)\right] \lesssim(130 \mathrm{GeV})^{2},
$$

where the first term in the r.h.s. corresponds to the tree-level prediction and the second term includes the one loop corrections due to the top and stop loops. Here, $m_{Z}, m_{t}, m_{\tilde{t}}$ are the $Z$-boson, the top and stop quark masses, respectively, $v=\sqrt{v_{1}^{2}+v_{2}^{2}}$ with $v_{i}$ the VEVs of the two higgses, $\tan \beta=v_{2} / v_{1}$, and $A_{t}$ the trilinear stop scalar coupling. Thus, a Higgs mass around $126 \mathrm{GeV}$ requires a heavy stop $m_{\tilde{t}} \simeq 3 \mathrm{TeV}$ for vanishing $A_{t}$, or $A_{t} \simeq 3 m_{\tilde{t}} \simeq 1.5 \mathrm{TeV}$ in the 'best' case. These values are obviously consistent with the present LHC bounds on supersymmetry searches, but they will certainly be probed in the next run at double energy. Theoretically, they imply a fine-tuning of the electroweak (EW) scale at the percent to per mille level. This fine-tuning can be alleviated in supersymmetric models beyond the MSSM, as discussed in the next session.

\section{MSSM Higgs sector with dimension-five and dimension-six operators}

Although extremely successful, the Standard Model or its supersymmetric version (MSSM) is not a fundamental theory, and this motivated the theoretical efforts to understand the nature of new physics beyond it. This search can be done using an effective field theory approach, in which the "new physics" is parametrised by effective operators. The power of this approach resides in arranging these operators in powers of $1 / M_{*}$ where $M_{*}$ is the scale of new physics that generated them. To improve the predictive power, one considers additional organising principles, such as: (i) symmetry constraints that these operators should respect, often inspired by phenomenology (for example: R-parity, lepton or baryon number conservation, etc). (ii) a truncation of the series of operators to a given order in the power of the inverse scale $1 / M_{*}$. The effective low-energy Lagrangian then takes the form

$$
\mathcal{L}=\mathcal{L}_{0}+\sum_{i, n} \frac{c_{n, i}}{M_{*}^{n}} O_{n, i}
$$

where $\mathcal{L}_{0}$ is the SM or the MSSM Lagrangian; $O_{n, i}$ is an operator of dimension $d=n+4$ with the index $i$ running over the set of operators of a given dimension; $c_{n, i}$ are some coefficients of order $O(1)$. This description is appropriate for scales $E$ which satisfy $E \ll M_{*}$. Constraints from phenomenology can then be used to set bounds on the scale of new physics $M_{*}$.

Regarding the origin of operators $O_{n, i}$, they can be generated classically or at the quantum level. At the classical level, this can happen by integration of some new massive states, via the equations of motion and one then generates an infinite series. This can happen even in 4D renormalisable theories; indeed, even though the low energy interaction looks nonrenormalisable, it may actually point to a renormalisable theory valid up to a much higher scale (a familiar example is the Fermi interaction). Such effective operators are also generated at the quantum level, for example following compactification of a higher dimensional theory, by the radiative corrections associated with momentum and winding modes of the compactification [1-5].

The effects of these operators on the low energy observables can be comparable to the radiative effects of light states in the SM/MSSM [6] and this shows the importance of their study. In the following we shall study these effects to the case of the MSSM Higgs sector with additional operators of dimensions $d=5$ and $d=6[6,7]$. In particular we show that the mass of lightest SM-like Higgs can easily be increased close to the observed value by new physics in the region of few TeV. We then discuss the nature of the "new physics" behind the effective operators. 


\subsection{MSSM Higgs sector with $d=5$ and $d=6$ operators}

In the leading order, new physics beyond the MSSM Higgs sector can manifest itself as operators of either $d=5[6,12-14]$ or $d=6[7,15]$ or both. If generated by the same new physics, by comparing $O\left(1 / M^{*}\right)$ and $O\left(1 / M_{*}^{2}\right)$ terms one can estimate when the series expansion in $1 / M_{*}$ breaks down. There is only one operator in the Higgs sector of dimension $d=5$ :

$$
\mathcal{L}_{1}=\frac{1}{M_{*}} \int d^{2} \theta \lambda_{H}^{\prime}(S)\left(H_{2} \cdot H_{1}\right)^{2}+h . c .=2 \zeta_{10}\left(h_{2} \cdot h_{1}\right)\left(h_{2} \cdot F_{1}+F_{2} \cdot h_{1}\right)+\zeta_{11} m_{0}\left(h_{2} \cdot h_{1}\right)^{2}+h \cdot c,
$$

where $\lambda_{H}^{\prime}(S) / M_{*}=\zeta_{10}+\zeta_{11} m_{0} \theta \theta$ and $\zeta_{10}, \zeta_{11} \sim 1 / M_{*}$. It can be generated by integrating out a massive gauge singlet or $S U(2)$ triplet. Indeed, in the MSSM with a massive gauge singlet, with an F-term of type $M_{*} \Sigma^{2}+\Sigma H_{1} \cdot H_{2}$, when integrating out $\Sigma$ generates $\mathcal{L}_{1}$. With the standard notation, here $S=m_{0} \theta^{2}$ is the spurion superfield and $m_{0}$ the supersymmetry breaking scale.

We assume that $m_{0} \ll M_{*}$, so that the effective approach is reliable. If this is not respected and the "new physics" is represented by "light" states (like the MSSM states), the $1 / M_{*}$ expansion is not reliable and one should work in a setup where these are not integrated out.

The list of $d=6$ operators is longer [16]:

$$
\begin{array}{ll}
O_{1}=\frac{1}{M_{*}^{2}} \int d^{4} \theta \mathcal{Z}_{1}\left(H_{1}^{\dagger} e^{V_{1}} H_{1}\right)^{2}, & O_{5}=\frac{1}{M_{*}^{2}} \int d^{4} \theta \mathcal{Z}_{5}\left(H_{1}^{\dagger} e^{V_{1}} H_{1}\right) H_{2} . H_{1}+\text { h.c. } \\
O_{2}=\frac{1}{M_{*}^{2}} \int d^{4} \theta \mathcal{Z}_{2}\left(H_{2}^{\dagger} e^{V_{2}} H_{2}\right)^{2}, & O_{6}=\frac{1}{M_{*}^{2}} \int d^{4} \theta \mathcal{Z}_{6}\left(H_{2}^{\dagger} e^{V_{2}} H_{2}\right) H_{2} . H_{1}+\text { h.c. } \\
O_{3}=\frac{1}{M_{*}^{2}} \int d^{4} \theta \mathcal{Z}_{3}\left(H_{1}^{\dagger} e^{V_{1}} H_{1}\right)\left(H_{2}^{\dagger} e^{V_{2}} H_{2}\right), & O_{7}=\frac{1}{M_{*}^{2}} \int d^{2} \theta \mathcal{Z}_{7} \operatorname{Tr} W^{\alpha} W_{\alpha}\left(H_{2} H_{1}\right)+\text { h.c. } \\
O_{4}=\frac{1}{M_{*}^{2}} \int d^{4} \theta \mathcal{Z}_{4}\left(H_{2} . H_{1}\right)\left(H_{2} . H_{1}\right)^{\dagger}, & O_{8}=\frac{1}{M_{*}^{2}} \int d^{4} \theta \mathcal{Z}_{8}\left(H_{2} H_{1}\right)^{2}+\text { h.c. }
\end{array}
$$

where $W^{\alpha}=(-1 / 4) \bar{D}^{2} e^{-V} D^{\alpha} e^{V}$ is the chiral field strength of $S U(2)_{L}$ or $U(1)_{Y}$ vector superfields $V_{w}$ and $V_{Y}$ respectively. Also $V_{1,2}=V_{w}^{a}\left(\sigma^{a} / 2\right)+(\mp 1 / 2) V_{Y}$ with the upper (minus) sign for $V_{1}$. Finally, the wavefunction coefficients are spurion dependent and have the structure

$$
\left(1 / M_{*}^{2}\right) \mathcal{Z}_{i}\left(S, S^{\dagger}\right)=\alpha_{i 0}+\alpha_{i 1} m_{0} \theta \theta+\alpha_{i 1}^{*} m_{0} \overline{\theta \theta}+\alpha_{i 2} m_{0}^{2} \theta \theta \overline{\theta \theta}, \quad \alpha_{i j} \sim 1 / M_{*}^{2} .
$$

Regarding the origin of these operators: $O_{1,2,3}$ can be generated in the MSSM by an additional, massive $U(1)^{\prime}$ gauge boson or $S U(2)$ triplets, when integrated out [12]. $O_{4}$ can be generated by a massive gauge singlet or $S U(2)$ triplet, while $O_{5,6}$ can be generated by a combination of $S U(2)$ doublets and massive gauge singlet. $O_{7}$ is essentially a threshold correction to the gauge coupling, with a moduli field replaced by the Higgs. $O_{8}$ exists only in non-susy case, but is generated when removing a $d=5$ derivative operator by field redefinitions [6], so we keep it.

\subsection{Higgs mass corrections from $d=5$ and $d=6$ operators.}

With the above set of independent, effective operators, one finds the scalar potential $V$ and its EW minimum which is perturbed by $O\left(1 / M_{*}^{2}\right)$ corrections from that of the MSSM. From $V$ one computes the mass of CP-odd/even Higgs fields:

$$
m_{A}^{2}=\left(m_{A}^{2}\right)_{\mathrm{MSSM}}-\frac{2 \zeta_{10} \mu_{0} v^{2}}{\sin 2 \beta}+2 m_{0} \zeta_{11} v^{2}+\delta m_{A}^{2}, \quad \delta m_{A}^{2}=O\left(1 / M_{*}^{2}\right)
$$


for the pseudoscalar Higgs, with $\left(m_{A}^{2}\right)_{\text {MSSM }}$ the MSSM value, with $\delta m_{A}^{2}$ due to $O\left(1 / M_{*}^{2}\right)$ corrections from $d=5$ and $d=6$ operators. For the CP-even Higgs one has [6, 12,14]

$$
\begin{aligned}
m_{h, H}^{2} & =\left(m_{h, H}^{2}\right)_{\mathrm{MSSM}} \\
& +\left(2 \zeta_{10} \mu_{0}\right) v^{2} \sin 2 \beta\left[1 \pm \frac{m_{A}^{2}+m_{Z}^{2}}{\sqrt{\tilde{w}}}\right]+\frac{\left(-2 \zeta_{11} m_{0}\right) v^{2}}{2}\left[1 \mp \frac{\left(m_{A}^{2}-m_{Z}^{2}\right) \cos ^{2} 2 \beta}{\sqrt{\tilde{w}}}\right] \\
& +\delta m_{h, H}^{2}, \quad \text { where } \quad \delta m_{h, H}^{2}=O\left(1 / M_{*}^{2}\right)
\end{aligned}
$$

The upper (lower) signs correspond to $h(H)$, and $\tilde{w} \equiv\left(m_{A}^{2}+m_{Z}^{2}\right)^{2}-4 m_{A}^{2} m_{Z}^{2} \cos ^{2} 2 \beta$. With this result one can show that the mass $m_{h}$ can be increased near the observed value, also with the help of quantum corrections [6, 12-14].

Regarding the $O\left(1 / M_{*}^{2}\right)$ corrections of $\delta m_{h, H}^{2}, \delta m_{A}^{2}$ and $\delta m_{h, H}^{2}$ of (6), (7), in the general case of including all operators and their associated supersymmetry breaking, they have a complicated form. Exact expressions can be found in [7, 15]. For most purposes, an expansion of these in $1 / \tan \beta$ is accurate enough. At large $\tan \beta, d=6$ operators bring corrections comparable to those of $d=5$ operators. The relative $\tan \beta$ enhancement of $O\left(1 / M_{*}^{2}\right)$ corrections compensates for the extra suppression that these have relative to $O\left(1 / M_{*}\right)$ operators (which involve both $h_{1}, h_{2}$ and are not enhanced in this limit). Note however that in some models only $d=6$ operators may be present, depending on the details of the "new physics" generating the effective operators.

Let us present the correction $O\left(1 / M^{2}\right)$ to $m_{h}^{2}$ for the case $m_{A}$ is kept fixed to an appropriate value. The result is, assuming $m_{A}>m_{Z}$, (otherwise $\delta m_{h}^{2}$ and $\delta m_{H}^{2}$ are exchanged):

$$
\begin{aligned}
\delta m_{h}^{2} & =-2 v^{2}\left[\alpha_{22} m_{0}^{2}+\left(\alpha_{30}+\alpha_{40}\right) \mu_{0}^{2}+2 \alpha_{61} m_{0} \mu_{0}-\alpha_{20} m_{Z}^{2}\right]-\left(2 \zeta_{10} \mu_{0}\right)^{2} v^{4}\left(m_{A}^{2}-m_{Z}^{2}\right)^{-1} \\
& +v^{2} \cot \beta\left[( m _ { A } ^ { 2 } - m _ { Z } ^ { 2 } ) ^ { - 1 } \left(4 m_{A}^{2}\left(\left(2 \alpha_{21}+\alpha_{31}+\alpha_{41}+2 \alpha_{81}\right) m_{0} \mu_{0}+\left(2 \alpha_{50}+\alpha_{60}\right) \mu_{0}^{2}+\alpha_{62} m_{0}^{2}\right)\right.\right. \\
& \left.\left.-\left(2 \alpha_{60}-3 \alpha_{70}\right) m_{A}^{2} m_{Z}^{2}-\left(2 \alpha_{60}+\alpha_{70}\right) m_{Z}^{4}\right)+8\left(m_{A}^{2}+m_{Z}^{2}\right)\left(\mu_{0} m_{0} \zeta_{10} \zeta_{11}\right) v^{2} /\left(m_{A}^{2}-m_{Z}^{2}\right)^{2}\right] \\
& +O\left(1 / \tan ^{2} \beta\right)
\end{aligned}
$$

The mass corrections in (8) must be added to the rhs of eq.(7) to obtain the full value of $m_{h}^{2}$. Together with (4), (5), these corrections identify the operators of $d=6$ with the largest contributions, which is important for model building beyond the MSSM Higgs sector. These operators are $O_{2,3,4}$ in the absence of supersymmetry breaking and $O_{2,6}$ when this is broken. It is preferable, however, to increase $m_{h}^{2}$ by supersymmetric rather than supersymmetry-breaking effects of the effective operators, because the latter are less under control in the effective approach; also, one would favour a supersymmetric solution to the fine-tuning problem associated with increasing the MSSM Higgs mass. Therefore $O_{2,3,4}$ are the leading operators, with the remark that $O_{2}$ has a smaller effect, of order $\left(m_{Z} / \mu_{0}\right)^{2}$ relative to $O_{3,4}$ (for similar $\alpha_{j 0}, j=2,3,4$ ). At smaller $\tan \beta, O_{5,6}$ can also give significant contributions, while $O_{7}$ has a relative suppression factor $\left(m_{Z} / \mu_{0}\right)^{2}$. Note that we kept all operators $O_{i}$ independent. By doing so, one can easily single out the individual contribution of each operator, which helps in model building, since not all operators are present in a specific model.

One limit to consider is that where the operators of $d=6$ have coefficients such that their contributions add up to maximise $\delta m_{h}^{2}$. Since $\alpha_{i j}$ are not known, one can choose:

$$
-\alpha_{22}=-\alpha_{61}=-\alpha_{30}=-\alpha_{40}=\alpha_{20}>0
$$

In this case, at large $\tan \beta$ :

$$
\delta m_{h}^{2} \approx 2 v^{2} \alpha_{20}\left[m_{0}^{2}+2 m_{0} \mu_{0}+2 \mu_{0}^{2}+m_{Z}^{2}\right]
$$


A simple numerical example is illustrative. For $m_{0}=1 \mathrm{TeV}, \mu_{0}=350 \mathrm{GeV}$, and with $v \approx 246 \mathrm{GeV}$, one has $\delta m_{h}^{2} \approx 2.36 \alpha_{20} \times 10^{11}(\mathrm{GeV})^{2}$. Assuming $M_{*}=10 \mathrm{TeV}$ and ignoring $d=5$ operators, with $\alpha_{20} \sim 1 / M_{*}^{2}$ and the MSSM value of $m_{h}$ taken to be its upper classical limit $m_{Z}$ (reached for large $\tan \beta$ ), we obtain an increase of $m_{h}$ from $d=6$ operators alone of about $\Delta m_{h}=12.15 \mathrm{GeV}$ to $m_{h} \approx 103 \mathrm{GeV}$. An increase of $\alpha_{20}$ by a factor of 2.5 to $\alpha_{20} \sim 2.5 / M_{*}^{2}$ would give $\Delta m_{h} \approx 28 \mathrm{GeV}$ to $m_{h} \approx 119.2 \mathrm{GeV}$, which is already above the LEP bound. Note that this increase is realised even for a scale $M_{*}$ of new physics beyond the LHC reach.

The above choice of $M_{*}=10 \mathrm{TeV}$ was partly motivated by the fine-tuning results [13] and on convergence grounds: the expansion parameter of our effective analysis is $m_{q} / M_{*}$ where $m_{q}$ is any scale of the theory, in particular it can be $m_{0}$. For a susy breaking scale $m_{0} \sim O(1) \mathrm{TeV}$ (say $m_{0}=3$ $\mathrm{TeV}$ ) and $c_{1,2}$ or $\alpha_{i j}$ of $\mathcal{Z}_{i}\left(S, S^{\dagger}\right)$ of order unity (say $c_{1,2}=2.5$ ) one has for $M_{*}=10 \mathrm{TeV}$ that $c_{1,2} m_{0} / M_{*}=0.75$ which is already close to unity, and at the limit of validity of the effective expansion in powers of $1 / M_{*}$. To conclude, even for a scale of "new physics" above the LHC reach, one can still classically increase $m_{h}$ to near the LHC measured value.

\section{Strings and extra dimensions}

The appropriate and most convenient framework for low energy supersymmetry and grand unification is the perturbative heterotic string. Indeed, in this theory, gravity and gauge interactions have the same origin, as massless modes of the closed heterotic string, and they are unified at the string scale $M_{s}$. As a result, the Planck mass is predicted to be proportional to $M_{s}$ :

$$
M_{P}=M_{s} / g
$$

where $g$ is the gauge coupling. In the simplest constructions all gauge couplings are the same at the string scale, given by the four-dimensional (4d) string coupling, and thus no grand unified group is needed for unification. In our conventions $\alpha_{\mathrm{GUT}}=g^{2} \simeq 0.04$, leading to a discrepancy between the string and grand unification scale $M_{\mathrm{GUT}}$ by almost two orders of magnitude. Explaining this gap introduces in general new parameters or a new scale, and the predictive power is essentially lost. This is the main defect of this framework, which remains though an open and interesting possibility.

The other other perturbative framework that has been studied extensively in the more recent years is type I string theory with D-branes. Unlike in the heterotic string, gauge and gravitational interactions have now different origin. The latter are described again by closed strings, while the former emerge as excitations of open strings with endpoints confined on D-branes [17]. This leads to a braneworld description of our universe, which should be localized on a hypersurface, i.e. a membrane extended in $p$ spatial dimensions, called $p$-brane (see Fig. 1). Closed strings propagate in all nine dimensions of string theory: in those extended along the $p$-brane, called parallel, as well as in the transverse ones. On the contrary, open strings are attached on the $p$-brane. Obviously, our $p$-brane world must have at least the three known dimensions of space. But it may contain more: the extra $d_{\|}=p-3$ parallel dimensions must have a finite size, in order to be unobservable at present energies, and can be as large as $\mathrm{TeV}^{-1} \sim 10^{-18} \mathrm{~m} \mathrm{[18]}$. On the other hand, transverse dimensions interact with us only gravitationally and experimental bounds are much weaker: their size should be less than about $0.1 \mathrm{~mm}$ [19]. In the following, I review the main properties and experimental signatures of low string scale models [20].

\subsection{Framework of low scale strings}

In type I theory, the different origin of gauge and gravitational interactions implies that the relation between the Planck and string scales is not linear as (11) of the heterotic string. The requirement that 


\section{EPJ Web of Conferences}

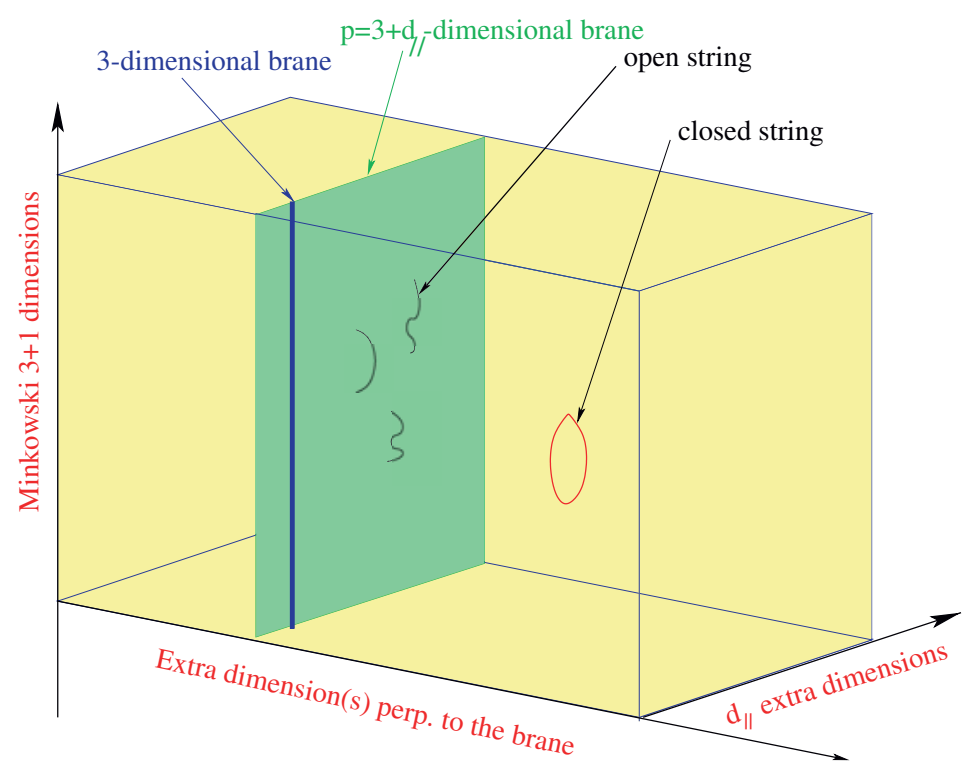

Figure 1. D-brane world universe in type I string framework.

string theory should be weakly coupled, constrain the size of all parallel dimensions to be of order of the string length, while transverse dimensions remain unrestricted. Assuming an isotropic transverse space of $n=9-p$ compact dimensions of common radius $R_{\perp}$, one finds:

$$
M_{P}^{2}=\frac{1}{g_{s}^{2}} M_{s}^{2+n} R_{\perp}^{n}, \quad g_{s} \simeq g^{2}
$$

where $g_{s}$ is the string coupling. It follows that the type I string scale can be chosen hierarchically smaller than the Planck mass [20,21] at the expense of introducing extra large transverse dimensions felt only by gravity, while keeping the string coupling small [20]. The weakness of $4 \mathrm{~d}$ gravity compared to gauge interactions (ratio $M_{W} / M_{P}$ ) is then attributed to the largeness of the transverse space $R_{\perp}$ compared to the string length $l_{s}=M_{s}^{-1}$.

An important property of these models is that gravity becomes effectively $(4+n)$-dimensional with a strength comparable to those of gauge interactions at the string scale. The first relation of Eq. (12) can be understood as a consequence of the $(4+n)$-dimensional Gauss law for gravity, with $M_{*}^{(4+n)}=M_{s}^{2+n} / g^{4}$ the effective scale of gravity in $4+n$ dimensions. Taking $M_{s} \simeq 1 \mathrm{TeV}$, one finds a size for the extra dimensions $R_{\perp}$ varying from $10^{8} \mathrm{~km}, .1 \mathrm{~mm}$, down to a Fermi for $n=1,2$, or 6 large dimensions, respectively. This shows that while $n=1$ is excluded, $n \geq 2$ is allowed by present experimental bounds on gravitational forces $[19,22]$. Thus, in these models, gravity appears to us very weak at macroscopic scales because its intensity is spread in the "hidden" extra dimensions. At distances shorter than $R_{\perp}$, it should deviate from Newton's law, which may be possible to explore in laboratory experiments. 


\subsection{Experimental implications in accelerators}

We now turn to the experimental predictions of TeV scale strings. Their main implications in particle accelerators are of four types, in correspondence with the four different sectors that are generally present:

1. New compactified parallel dimensions; In this case $R M_{s} \gtrsim 1$, and the associated compactification scale $R_{\|}^{-1}$ would be the first scale of new physics that should be found increasing the beam energy $[18,23]$. The main consequence is the existence of KK excitations for all SM particles that propagate along the extra parallel dimensions. These can be produced on-shell at LHC as new resonances [24].

2. New extra large transverse dimensions and low scale quantum gravity,. The main experimental signal is gravitational radiation in the bulk from any physical process on the world-brane [25].

3. Genuine string and quantum gravity effects. Direct production of string resonances in hadron colliders leads generically to a universal deviation from Standard Model in jet distribution [26]. In particular, the first Regge excitation of the gluon has spin 2 and a width an order of magnitude lower than the string scale, leading to a characteristic peak in dijet production; similarly, the first excitations of quarks have spin $3 / 2$.

4. Extra $U(1)$ 's arising generically in D-brane models as part of unitary gauge group factors. They obtain in general masses due to four- or higher-dimensional anomalies, via the so-called GreenSchwarz anomaly cancellation mechanism involving axionic fields from the closed string sector. The resulting masses are therefore suppressed by a loop factor compared to the string scale. From the low energy point of view, they gauge global symmetries of the Standard Model, such as the baryon and lepton number. An important property of the anomaly cancellation mechanism is that the anomalous $U(1)$ gauge bosons acquire masses leaving behind the corresponding global symmetries unbroken in perturbation theory. Thus, this is a way to guarantee proton stability (from unbroken baryon number) and avoid large Majorana neutrino masses (from unbroken lepton number) due to dimension-5 operators involving two higgses and two leptons that are suppressed only by the TeV string scale. Such extra $U(1)$ s have interesting properties and distinct experimental signatures [27-29].

5. Concerning possible micro-black hole production, note that a string size black hole has a horizon radius $r_{H} \sim 1$ in string units, while the Newton's constant behaves as $G_{N} \sim g_{s}^{2}$. It follows that the mass of a $d$-dimensional black hole is [30]: $M_{\mathrm{BH}} \sim r_{H}^{d / 2-1} / G_{N} \simeq 1 / g_{s}^{2}$. Using the value of the SM gauge couplings $g_{s} \simeq g^{2} \sim 0.1$, one finds that the energy threshold $M_{\mathrm{BH}}$ of microblack hole production is about four orders of magnitude higher than the string scale, implying that one would produce $10^{4}$ string states before reaching $M_{\mathrm{BH}}$.

\subsection{Electroweak symmetry breaking}

Non-supersymmetric $\mathrm{TeV}$ strings offer also a framework to realize gauge symmetry breaking radiatively. Indeed, from the effective field theory point of view, one expects quadratically divergent oneloop contributions to the masses of scalar fields. The divergences are cut off by $M_{s}$ and if the corrections are negative, they can induce electroweak symmetry breaking and explain the mild hierarchy between the weak and a string scale at a few $\mathrm{TeV}$, in terms of a loop factor [31]. More precisely, in the minimal case of one Higgs doublet $H$, the scalar potential is:

$$
V=\lambda\left(H^{\dagger} H\right)^{2}+\mu^{2}\left(H^{\dagger} H\right),
$$


where $\lambda$ arises at tree-level. Moreover, in any model where the Higgs field comes from an open string with both ends fixed on the same brane stack, it is given by an appropriate truncation of a supersymmetric theory. On the other hand, $\mu^{2}$ is generated at one loop:

$$
\mu^{2}=-\varepsilon^{2} g^{2} M_{s}^{2}
$$

where $\varepsilon$ is a loop factor that can be estimated from a toy model computation and varies in the region $\epsilon \sim 10^{-1}-10^{-3}$.

Indeed, consider for illustration a simple case where the whole one-loop effective potential of a scalar field can be computed. We assume for instance one extra dimension compactified on a circle of radius $R>1$ (in string units). An interesting situation is provided by a class of models where a non-vanishing VEV for a scalar (Higgs) field $\phi$ results in shifting the mass of each KK excitation by a constant $a(\phi)$ :

$$
M_{m}^{2}=\left(\frac{m+a(\phi)}{R}\right)^{2},
$$

with $m$ the KK integer momentum number. Such mass shifts arise for instance in the presence of a Wilson line, $a=q \oint \frac{d y}{2 \pi} A$, where $A$ is the internal component of a gauge field and $q$ the charge of a given state under the corresponding generator. A straightforward computation shows that the $\phi$-dependent part of the one-loop effective potential is given by [32]:

$$
V_{e f f}=-\operatorname{Tr}(-)^{F} \frac{R}{32 \pi^{3 / 2}} \sum_{n} e^{2 \pi i n a} \int_{0}^{\infty} d l l^{3 / 2} f_{s}(l) e^{-\pi^{2} n^{2} R^{2} l}
$$

where $F=0,1$ for bosons and fermions, respectively. We have included a regulating function $f_{s}(l)$ which contains for example the effects of string oscillators. To understand its role we will consider the two limits $R>>1$ and $R<<1$. In the first case only the $l \rightarrow 0$ region contributes to the integral. This means that the effective potential receives sizable contributions only from the infrared (field theory) degrees of freedom. In this limit we would have $f_{s}(l) \rightarrow 1$. For example, in the string model considered in [31]:

$$
f_{s}(l)=\left[\frac{1}{4 l} \frac{\theta_{2}}{\eta^{3}}\left(i l+\frac{1}{2}\right)\right]^{4} \rightarrow 1 \quad \text { for } \quad l \rightarrow 0,
$$

and the field theory result is finite and can be explicitly computed. As a result of the Taylor expansion around $a=0$, we are able to extract the one-loop contribution to the coefficient of the term of the potential quadratic in the Higgs field. It is given by a loop factor times the compactification scale [32]. One thus obtains $\mu^{2} \sim g^{2} / R^{2}$ up to a proportionality constant which is calculable in the effective field theory. On the other hand, if we consider $R \rightarrow 0$, which by $T$-duality corresponds to taking the extra dimension as transverse and very large, the one-loop effective potential receives contributions from the whole tower of string oscillators as appearing in $f_{s}(l)$, leading to squared masses given by a loop factor times $M_{s}^{2}$, according to eq. (14).

More precisely, from the expression (16), one finds:

$$
\varepsilon^{2}(R)=\frac{1}{2 \pi^{2}} \int_{0}^{\infty} \frac{d l}{(2 l)^{5 / 2}} \frac{\theta_{2}^{4}}{4 \eta^{12}}\left(i l+\frac{1}{2}\right) R^{3} \sum_{n} n^{2} e^{-2 \pi n^{2} R^{2} l} .
$$

For the asymptotic value $R \rightarrow 0$ (corresponding upon T-duality to a large transverse dimension of radius $1 / R), \varepsilon(0) \simeq 0.14$, and the effective cut-off for the mass term is $M_{s}$, as can be seen from Eq. (14). At large $R, \mu^{2}(R)$ falls off as $1 / R^{2}$, which is the effective cut-off in the limit $R \rightarrow \infty$, 
as we argued above, in agreement with field theory results in the presence of a compactified extra dimension.In fact, in the limit $R \rightarrow \infty$, an analytic approximation to $\varepsilon(R)$ gives:

$$
\varepsilon(R) \simeq \frac{\varepsilon_{\infty}}{M_{s} R}, \quad \varepsilon_{\infty}^{2}=\frac{3 \zeta(5)}{4 \pi^{4}} \simeq 0.008 .
$$

The potential (13) has the usual minimum, given by the VEV of the neutral component of the Higgs doublet $v=\sqrt{-\mu^{2} / \lambda}$. Furthermore, from (14), one can compute $M_{s}$ in terms of the Higgs mass $m_{H}^{2}=-2 \mu^{2}: M_{s}=\frac{m_{H}}{\sqrt{2} g \varepsilon}$, yielding naturally values in the TeV range.

\section{Acknowledgments}

Work supported in part by the European Commission under the ERC Advanced Grant 226371.

\section{References}

\section{References}

[1] D. M. Ghilencea and H. M. Lee, JHEP 0509 (2005) 024.

[2] D. M. Ghilencea, Phys. Rev. D 70 (2004) 045018.

[3] S. Groot Nibbelink, M. Hillenbach, Phys. Lett. B 616 (2005) 125.

[4] S. Groot Nibbelink, M. Hillenbach, Nucl.Phys. B 748 (2006) 60.

[5] J. F. Oliver, J. Papavassiliou and A. Santamaria, Phys. Rev. D 67 (2003) 125004.

[6] I. Antoniadis, E. Dudas, D. Ghilencea, P. Tziveloglou, Nucl. Phys. B 808 (2009) 155.

[7] I. Antoniadis, E. Dudas, D. Ghilencea, P. Tziveloglou, Nucl. Phys. B 831 (2010) 133.

[8] G. F. Giudice, C. Grojean, A. Pomarol and R. Rattazzi, JHEP 0706 (2007) 045.

[9] S. Cassel, D. M. Ghilencea, G. G. Ross, Nucl. Phys. B 835 (2010) 110.

[10] S. Cassel, D. M. Ghilencea and G. G. Ross, Phys. Lett. B 687 (2010) 214.

[11] R. Barate et al. [LEP Working Group for Higgs boson searches and ALEPH, DELPHI, L3, OPAL Collaborations, Phys. Lett. B 565 (2003) 61; S. Schael et al. ALEPH, DELPHI, L3, OPAL and LEP Working Group for Higgs Boson Searches, Eur. Phys. J. C47(2006)547

[12] M. Dine, N. Seiberg, S. Thomas, Phys. Rev. D 76 (2007) 095004.

[13] S. Cassel, D. Ghilencea, G. G. Ross, Nucl. Phys. B 825 (2010) 203.

[14] K. Blum, C. Delaunay and Y. Hochberg, arXiv:0905.1701 [hep-ph].

[15] M. Carena, K. Kong, E. Ponton and J. Zurita, arXiv:0909.5434 [hep-ph].

M. Carena, E. Ponton and J. Zurita, arXiv:1005.4887 [hep-ph].

[16] D. Piriz and J. Wudka, Phys. Rev. D 56 (1997) 4170.

A. Brignole, J. A. Casas, J. R. Espinosa and I. Navarro,

[17] C. Angelantonj and A. Sagnotti, Phys. Rept. 371 (2002) 1 [Erratum-ibid. 376 (2003) 339] [arXiv:hep-th/0204089].

[18] I. Antoniadis, Phys. Lett. B 246 (1990) 377.

[19] D. J. Kapner, T. S. Cook, E. G. Adelberger, J. H. Gundlach, B. R. Heckel, C. D. Hoyle and H. E. Swanson, Phys. Rev. Lett. 98 (2007) 021101.

[20] N. Arkani-Hamed, S. Dimopoulos and G. R. Dvali, Phys. Lett. B 429 (1998) 263 [arXiv:hepph/9803315]; I. Antoniadis, N. Arkani-Hamed, S. Dimopoulos and G. R. Dvali, Phys. Lett. B 436 (1998) 257 [arXiv:hep-ph/9804398]. 
[21] J. D. Lykken, Phys. Rev. D 54 (1996) 3693 [arXiv:hep-th/9603133].

[22] J.C. Long and J.C. Price, Comptes Rendus Physique 4 (2003) 337; R.S. Decca, D. Lopez, H.B. Chan, E. Fischbach, D.E. Krause and C.R. Jamell, Phys. Rev. Lett. 94 (2005) 240401; R.S. Decca et al., arXiv:0706.3283 [hep-ph]; S.J. Smullin, A.A. Geraci, D.M. Weld, J. Chiaverini, S. Holmes and A. Kapitulnik, arXiv:hep-ph/0508204; H. Abele, S. Haeßler and A. Westphal, in 271th WE-Heraeus-Seminar, Bad Honnef (2002).

[23] I. Antoniadis and K. Benakli, Phys. Lett. B 326 (1994) 69.

[24] I. Antoniadis, K. Benakli and M. Quirós, Phys. Lett. B 331 (1994) 313 and Phys. Lett. B 460 (1999) 176; P. Nath, Y. Yamada and M. Yamaguchi, Phys. Lett. B 466 (1999) 100 T. G. Rizzo and J. D. Wells, Phys. Rev. D 61 (2000) 016007; T. G. Rizzo, Phys. Rev. D 61 (2000) 055005; A. De Rujula, A. Donini, M. B. Gavela and S. Rigolin, Phys. Lett. B 482 (2000) 195.

[25] G.F. Giudice, R. Rattazzi and J.D. Wells, Nucl. Phys. B 544 (1999) 3; E.A. Mirabelli, M. Perelstein and M.E. Peskin, Phys. Rev. Lett. 82 (1999) 2236; T. Han, J.D. Lykken and R. Zhang, Phys. Rev. D 59 (1999) 105006; K. Cheung, W.-Y. Keung, Phys. Rev. D 60 (1999) 112003; C. Balázs et al., Phys. Rev. Lett. 83 (1999) 2112; J.L. Hewett, Phys. Rev. Lett. 82 (1999) 4765.

[26] L.A. Anchordoqui, H. Goldberg, D. Lust, S. Nawata, S. Stieberger and T.R. Taylor, Phys. Rev. Lett. 101 (2008) 241803 [arXiv:0808.0497 [hep-ph]].

[27] I. Antoniadis, E. Kiritsis and T. N. Tomaras, Phys. Lett. B 486, 186 (2000) [arXiv:hepph/0004214]; I. Antoniadis, E. Kiritsis and J. Rizos, Nucl. Phys. B 637, 92 (2002) [arXiv:hepth/0204153]; I. Antoniadis, E. Kiritsis, J. Rizos and T. N. Tomaras, Nucl. Phys. B 660, 81 (2003) [arXiv:hep-th/0210263].

[28] G. Shiu and S.-H. H. Tye, Phys. Rev. D 58 (1998) 106007; Z. Kakushadze and S.-H. H. Tye, Nucl. Phys. B 548 (1999) 180; L. E. Ibáñez, C. Muñoz and S. Rigolin, Nucl. Phys. B 553 (1999) 43.

[29] L. A. Anchordoqui, I. Antoniadis, H. Goldberg, X. Huang, D. Lust and T. R. Taylor, Phys. Rev. D 85 (2012) 086003 [arXiv:1107.4309 [hep-ph]].

[30] G.T. Horowitz and J. Polchinski, Phys. Rev. D 55 (1997) 6189.

[31] I. Antoniadis, K. Benakli and M. Quirós, Nucl. Phys. B 583 (2000) 35.

[32] I. Antoniadis, K. Benakli and M. Quiros, New Jour. Phys. 3 (2001) 20. 